\title{
Spectral modulation of non-Galactic plane Gamma-ray pulsars due to photon-ALPs mixing in Galactic magnetic field.
}

\author{
Jhilik Majumdar $^{1}$, Francesca Calore ${ }^{2}$, Dieter Horns ${ }^{1}$ \\ ${ }^{1}$ Institute for Experimentalphysics, University of Hamburg. \\ ${ }^{2}$ Laboratoire d'Annecy-le-Vieux de Physique Théorique, CNRS. \\ E-mail: Jhilik.majumdaredesy.de
}

\begin{abstract}
Axion like particles (ALPs) are fundamental pseudo scalar particles with properties similar to Axions that have been invoked to solve the strong CP problem in Quantum Chromodynamics. ALPs can oscillate into photons and vice versa in the presence of an external magnetic field. This oscillation of Photon and ALPs could have important implications for astronomical observations, i.e. a characteristic energy dependent attenuation in Gamma ray spectra for astrophysical sources. Here we have revisited the opportunity to search Photon-ALPs coupling in the disappearance channel. We use nine years of Fermi Pass 8 data of a selection of non-Galactic plane Gamma-ray source candidates and study the modulation in the spectra in accordance with Photon-ALPs mixing and estimate best fit values of the parameters i.e. Photon-ALPs coupling constant $\left(g_{\alpha \gamma \gamma}\right)$ and ALPs mass $\left(m_{\alpha}\right)$. For the magnetic field we assume large scale galactic magnetic field models based on Faraday rotation measurements. We find consistent evidence with our prevoius analysis of objects located in the Galactic plane for a modulation of the spectra investigated at the level of 3.66 standard deviations . In the framework of ALPS/photon oscillation, the resulting parameters strongly depend upon the chosen magnetic field model, but seem to be within reach of new experiments (e.g. IAXO).
\end{abstract}

7th Fermi Symposium 2017

15-20 October 2017

Garmisch-Partenkirchen, Germany 


\section{Introduction}

The probable solution of strong CP problem in Quantum Chromodynamics has been proposed by Pecci \& Quinn postulating a global U(1) symmetry that is spontaneously broken at large energy scale [1]. Later on Weinberg and Wilczek showed that from this spontaneously broken PQ symmetry, a new pseudo NambuGoldstone boson comes into existance, which is also named as axion [2]. As this pseudo Nambu-Goldstone boson have a small mass due to non-perturbative effects or explicit symmetry breaking, they could be nice cold dark matter candidates [3]. Because of low-mass, axions have extremely low decay rates and they hardly make weak interactions with baryonic matter. On the other hand, Axion like particles(ALPs) with even smaller masses may exist and could be detected indirectly by astrophysical observations [4]. ALPs have a nice property that they can oscillate into photons or vice-versa in the presence of magnetic fields [5]. Photonaxion conversion induced by intergalactic magnetic fields causes an apparent attenuation in the photon flux of distant sources, depending on the distance of the source, the energy considered and also depending on the tranversal magnetic field along the line of sight [5]. A generic feature of axion models is the CP-conserving two-photon coupling, so that the axion-photon interaction is:

$$
\mathscr{L} \supset-\frac{1}{4} g_{\alpha \gamma \gamma} F_{\mu \nu} \tilde{F}^{\mu v} a=g_{\alpha \gamma \gamma} \vec{E} \cdot \vec{B} a,
$$

where $a$ is the axion-like field with mass $m_{a}, F_{\mu v}$ is the electromagnetic field-strength tensor and $\tilde{F}^{\mu v}$ is its dual field, $g_{\alpha \gamma \gamma}$ is the ALPs-photon coupling. Photons, while travelling across the external magnetic field, oscillate with the ALPs state. If the condition, $g_{\alpha \gamma \gamma} B d \ll 1$ holds true, the probability of the conversion at a distance $d$ is [6]:

$$
P_{\gamma \rightarrow a}=\frac{g_{\alpha \gamma \gamma}^{2}}{8}\left(\left|\int_{0}^{d} d z^{\prime} e^{2 \pi i z^{\prime} / l_{0}} B_{x}\left(x, y, z^{\prime}\right)\right|^{2}+\left|\int_{0}^{d} d z^{\prime} e^{2 \pi i z^{\prime} / l_{0}} B_{y}\left(x, y, z^{\prime}\right)\right|^{2}\right),
$$

Where, $g_{\alpha \gamma \gamma}$ has the dimension of (Energy) $)^{-1}$ and the parameters are in natural unit. Here, $l_{0}$ is the oscillation length of Photon-ALPs propagation. We are considering the sources with a distance in kpc range, Galactic magnetic field in $\mu G$; hence, the photon energy range we take from $100 \mathrm{MeV}$ to $300 \mathrm{GeV}$.

\section{Galactic Magnetic Field}

Best constraints for large scale Galactic Magnetic Field (GMF) are Faraday rotation measures, polarised synchrotron radiation and also the polarized dust emission from spinning elliptical dust grains. In our analysis we have taken into consideration one of the most comprehensive GMF model: Jansson \& Farrar model (2012) [7]. This model consists of three components: Disk component, Halo component and Out of plane component.The disk component is partially based on the structure of the NE2001 thermal electron density model [8]. The disk field is constrained to the $\mathrm{x}-\mathrm{y}$ plane and defined for Galactocentric radius from $3 \mathrm{kpc}$ to $20 \mathrm{kpc}$ with a 'molecular ring' and eight logarithmic spiral regions. The halo field has a purely toroidal component and separate field amplitudes in the north and south. The out of the plane component, formerly known as X component, is to be asymmetric and poloidal. The components of this large scale magnetic field has been updated with the polarized synchrotron and dust emission data measured with the Planck satellite [9].

\section{Source selection}

In the present work we use gamma-ray data from the Fermi-LAT [10].We have chosen a list of twelve bright gamma-ray pulsars with galactic latitude more than 10 degrees, listed in table 1 . In order to estimate systematic uncertainties on the observed spectrum we use as expected the Vela pulsar [11]. This pulsar is 

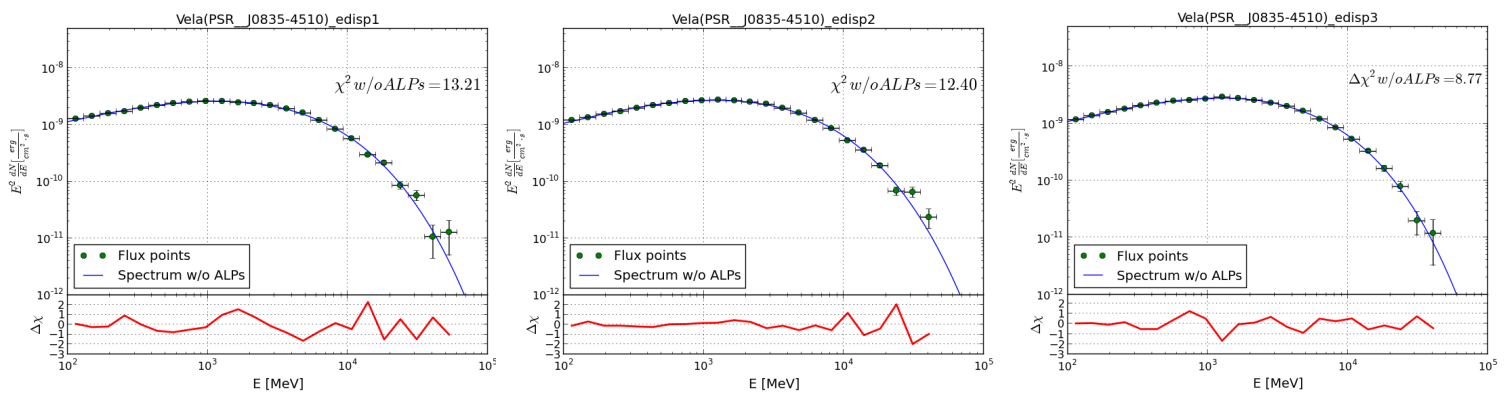

Figure 1: A fit of the phase averaged Vela spectrum with a power-law with exponential cut-off model. The $\chi^{2}$ fit to the differential flux points produces acceptable values under the assumption of a fractional systematic flux error of $2.4 \%$ over the entire energy range, separately shown for EDISP1, EDISP2, EDISP3.

Table 1: Gamma-ray pulsar selection list.

\begin{tabular}{|c|c|c|c|c|c|}
\hline Pulsar name & $\begin{array}{c}\text { Flux 0.1-300 GeV } \\
(\text { in photons } \\
\left.c^{-2} s^{-1}\right)\end{array}$ & $\begin{array}{c}\text { Flux } \\
\text { Error } \\
\pm\end{array}$ & $\begin{array}{c}\text { spectral } \\
\text { index }\end{array}$ & $\begin{array}{c}\text { Cut-off Energy } \\
\text { (in MeV) }\end{array}$ & $\begin{array}{c}\text { distance } \\
\text { (in kpc) }\end{array}$ \\
\hline J0007+7302 & $6.48 \mathrm{e}-08$ & $5.72 \mathrm{e}-10$ & 1.20 & 5298.87 & not known \\
$\mathrm{J} 0030+0451$ & $9.82 \mathrm{e}-09$ & $2.75 \mathrm{e}-10$ & 1.28 & 1739.96 & 0.36 \\
$\mathrm{~J} 0614-3329$ & $1.74 \mathrm{e}-08$ & $3.57 \mathrm{e}-10$ & 1.36 & 4462.26 & 0.62 \\
$\mathrm{~J} 1231-1411$ & $1.77 \mathrm{e}-08$ & $3.78 \mathrm{e}-10$ & 1.18 & 2286.05 & 0.42 \\
$\mathrm{~J} 1311-3430$ & $7.54 \mathrm{e}-09$ & $2.47 \mathrm{e}-10$ & 1.90 & 4922.37 & 2.43 \\
$\mathrm{~J} 1836+5925$ & $9.93 \mathrm{e}-08$ & $6.53 \mathrm{e}-09$ & 0.88 & 2011.65 & not known \\
$\mathrm{J} 2055+2539$ & $9.03 \mathrm{e}-09$ & $2.78 \mathrm{e}-10$ & 1.04 & 1175.53 & not known \\
$\mathrm{J} 2124-3358$ & $7.47 \mathrm{e}-09$ & $2.58 \mathrm{e}-10$ & 0.89 & 1677.98 & 0.41 \\
$\mathrm{~J} 2214+3000$ & $5.64 \mathrm{e}-09$ & $2.17 \mathrm{e}-10$ & 1.20 & 1667.98 & 0.60 \\
$\mathrm{~J} 2229-0833$ & $2.94 \mathrm{e}-08$ & $4.56 \mathrm{e}-10$ & 1.80 & 2396.16 & not known \\
$\mathrm{J} 2241-5237$ & $5.21 \mathrm{e}-09$ & $2.03 \mathrm{e}-10$ & 1.25 & 2910.19 & not known \\
$\mathrm{J} 2302+4443$ & $6.53 \mathrm{e}-09$ & $2.34 \mathrm{e}-10$ & 1.19 & 2762.43 & not known \\
\hline
\end{tabular}

very close; the spectrum is very well measured and does not show any spectral distortion. We use a similar technique that has been done by the Fermi Collaboration to derive the systematics using Fermi-LAT Pass 7 data [11].

\section{Analysis}

We use eight and half years of Fermi-LAT Pass 8 data with P8R2 SOURCE V6 IRFs of twelve bright pulsar candidates, i.e. PSR J0007+7302 et. al and Vela. Pass 8 data has an improved angular resolution, a broader energy range, larger effective area, as well as reduced uncertainties in the instrumental response functions [11]. For spectral modelling of Fermi-LAT sources Enrico binned likelihood optimization technique[12] is performed for 25 energy bins. All of the pulsar spectrum is modelled by a power law with 
exponential cutoff:

$$
\frac{d N}{d E}=N_{0}\left(\frac{E}{E_{0}}\right)^{-\Gamma} \exp \left(-\frac{E}{E_{\mathrm{cut}}}\right)
$$

For Vela we use a power law with sub exponential cutoff :

$$
\frac{d N}{d E}=N_{0}\left(\frac{E}{E_{0}}\right)^{-\Gamma_{1}} \exp \left[\left(-\frac{E}{E_{\text {cut }}}\right)^{\Gamma_{2}}\right]
$$

We perform a fit to the data, minimising the $\chi^{2}$ function which provides a measure of goodness of fit [13] [14]. We have checked that the $\log \left(\right.$ likelihood) has a parabolic pattern and thus a $\chi^{2}$ analysis is appropriate. We derive the energy dispersion matrix $\left(D_{k k_{p}}\right)$ for one energy dispersion event type (EDISP) via the transformation of the number of counts in true energy of a particular energy bin to the number of counts in that bin of reconstructed energy (see Fig. 1) and we fully take it into account in the fit. We investigate the signature of photon-ALPs oscillations, including the effect of oscillations in the predicted spectra:

$$
\left(\frac{d N}{d E}\right)_{\text {fit }}=D_{k k_{p}} \cdot\left(1-P_{\gamma \rightarrow a}\left(E, g_{a \gamma \gamma}, m_{a}, d\right)\right) \cdot\left(\frac{d N}{d E}\right)
$$

Systematic uncertainties of Vela: For P8R2_SOURCE_V6 event class, systematic uncertainties in effective collection area are derived to be about $2.4 \%$ for EDISP1, EDISP2, EDISP3 event types for Vela considering the energy range from $100 \mathrm{MeV}$ to $300 \mathrm{GeV}$ (see Fig. 3). We follow the same technique as Fermi collaboration [11] and do in such a way so that the $\chi^{2}$ per degrees of freedom(dof) we get $\sim 1$ and it's an acceptable fit. Systemaics has been imposed in chanel to energy for global fitting and it effects to reduce the $\chi^{2}$ values for individual pulsars.
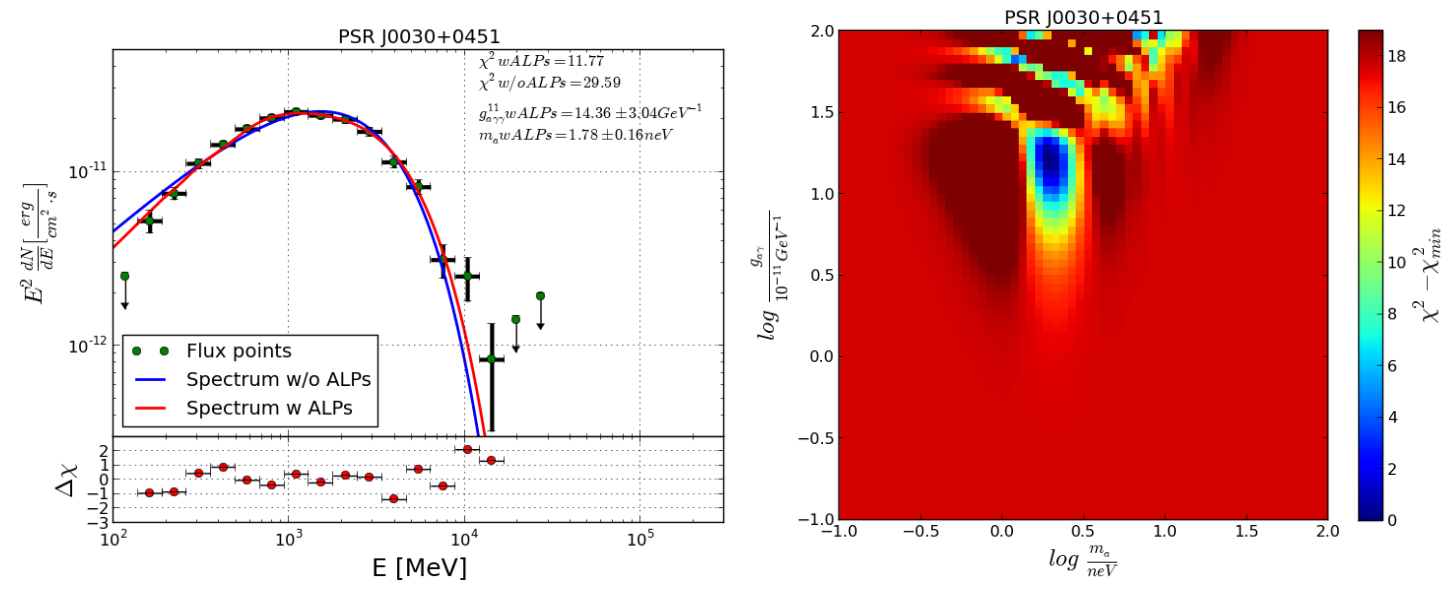

Figure 2: Left panel: Best-fit model of the spectrum of PSR J0030+0451 3651. Right panel: The $\chi^{2}$ scan as function of photon-ALPs coupling and ALPs mass.

\section{Result}

Apparent suppression in energy spectrum has been observed in case of Pulsar spectrum due to photonALPs mixing in the galactic magnetic field. The spectral feature in disappearance channel generally depends on the distance to the source and transversal magnetic field along the line of sight. We have seen the spectral 


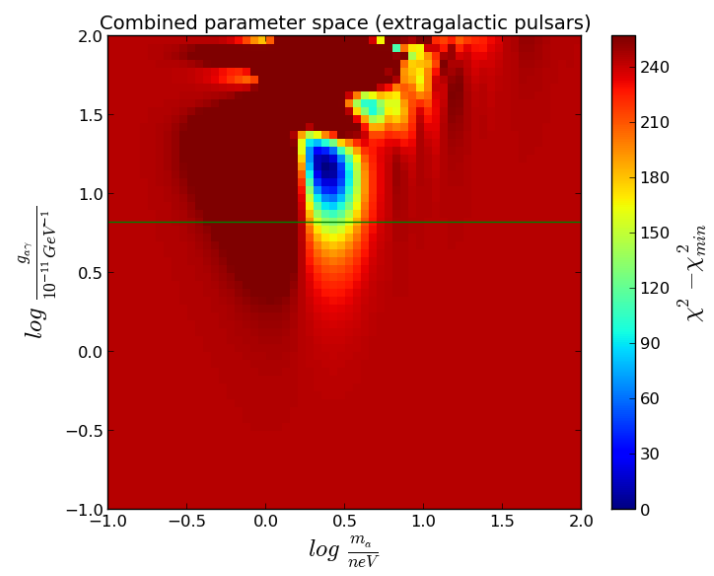

Figure 3: Combined $\chi^{2}$ scan as function of photon-ALPs coupling and ALPs mass.

modulation of the gamma-ray pulsar candidates and it would be explaied by photon-ALPs scenario. In case of PSR J0030+0451, the photon-ALPs coupling constant ands ALPs mass we get respectively $14.36 \times$ $10^{-11} \mathrm{GeV}^{-1}$ and $1.78 \mathrm{neV}$ (see Fig. 2). The photon-ALPs mixing phenomena makes a notable improvement in the $\chi^{2}$ value of the fitted spectrumreducing $\chi^{2}$ value by 17 from non-ALPs mixing to ALPs mixing case. We can say the fit results with ALPs parameters is a good fit as the $\chi^{2}$ per dof is $\sim 1$ for all the pulsar spectrum. In the fits we also include systematic uncertainties as derived from the Vela pulsar analysis as well as the energy dispersion. Fig. 2(right panel) depicts the blue stripes that corresponds to the photonALPs oscillation in tranversal magnetic field. The significance of our result for PSR J0030+0451 has been estimated by F-test $3.66 \sigma$. We make a combined parameter space for all the 12 pulsar candidates (see Fig. 3) and derive the best parameter space for photon-ALPs coupling constant and ALPs mass. PhotonALPs coupling constant $\left(g_{\alpha \gamma \gamma}\right)$ we get $14.12 \times 10^{-11} \mathrm{GeV}^{-1}$ and ALPs mass $\left(m_{\alpha}\right)$ we calculate $2.23 \mathrm{neV}$. The irregularities in pulsar spectrum can be explained by detailed analysis of photon-ALPs mixing in the galactic magnetic field, but we should be very cautious to choose the magnetic field model as the mixing parameters are very much model dependent.

\section{References}

[1] R. Peccei, HR. Quinn, CP conservation in the presence of Pseudoparticles, Phys. Rev. Lett. 38:1440 (1977).

[2] S. Wienburg, A New Light Boson? Phys. Rev. Lett. 40:223 (1978).

[3] J. Barranco, A. Carrillo Monteverde, D. Delepine, The axion-photon interaction and gamma ray signals of dark matter, Journal of Physics: Conference Series, Volume 485, conference 1.

[4] D.S. Gorbunov, G.G. Raffelt, D.V. Semikoz, Axion-like particles as ultra high energy cosmic rays? Phys. Rev. D. 64.096005.

[5] A. De Angelis, G. Galanti, M. Roncadelli, Importance of axion-like particles for very-high-energy astrophysics, Phys. Rev. D. 84.105030.

[6] A. Mirizzi, et al., Signatures of axion-like particles in the spectra of TeV gamma-ray sources, arXiv:0704.3044. 
[7] R. Jansson, G. R. Farrar, A New Model of the Galactic Magnetic Field, arXiv:1204.3662.

[8] X. Sun, and W. Reich, The Galactic halo magnetic field revisited, arXiv:1010.4394.

[9] R. Adam, et al., Planck intermediate results. XLII. Large-scale Galactic magnetic fields, arXiv:1601.00546.

[10] The Fermi-LAT Collaboration, Fermi Large Area Telescope Third Source Catalog, arXiv:1501.02003.

[11] M. Ackermann, et al., The Fermi Large Area Telescope On Orbit: Event Classification, Instrument Response Functions, and Calibration, arXiv:1206.1896.

[12] D.A. Sanchez, C. Deil, Enrico : a Python package to simplify Fermi-LAT analysis , arXiv:1307.4534.

[13] The Fermi LAT collaboration: M. Ackermann, et al., Detection of the Characteristic Pion-Decay Signature in Supernova Remnants. Science, 339(6121):807-11.

[14] T. Jogler, S. Funk, Revealing W51C as a cosmic ray source using Fermi-LAT data, The Astrophysical Journal, Volume 816, Number 2. 